\title{
THE NUMBER OF SOLUTIONS TO AN EQUATION ARISING FROM A PROBLEM ON LATIN SQUARES
}

\author{
I. P. GOULDEN and S. A. VANSTONE \\ (Received 13 October 1981; revised 3 March 1982) \\ Communicated by W. D. Wallis
}

\begin{abstract}
A recent article of $\mathrm{G}$. Chang shows that an $n \times n$ partial latin square with prescribed diagonal can always be embedded in an $n \times n$ latin square except in one obvious case where it cannot be done. Chang's proof is to show that the symbols of the partial latin square can be assigned the elements of the additive abelian group $\mathbf{Z}_{n}$ so that the diagonal elements of the square sum to zero. A theorem of M. Halls then shows this to be embeddable in the operation table of the group. In this paper, we show that when $n$ is a prime one can determine exactly the number of distinct ways in which this assignment can be made. The proof uses some graph theoretic techniques.
\end{abstract}

1980 Mathematics subject classification (Amer. Math. Soc.): 05 B 15.

\section{Introduction}

An $n \times n$ array $A$ defined on an $n$-set $V$ is called a partial latin square if each cell of $A$ is either empty or contains an element of $V$ and every element of $V$ is contained in at most one cell of each row and column of $A$. The following problem was recently solved by $\mathrm{G}$. Chang [2].

An $n \times n$ partial latin square in which only the cells on the main diagonal and only those cells contain elements of $V$ (a partial latin square with prescribed diagonal) can always be completed to an $n \times n$ latin square except in the case where the elements on the main diagonal consist of exactly 2 distinct symbols and one of these symbols occurs precisely once.

\footnotetext{
Copyright Australian Mathematical Society 1983
} 
The proof given by Chang appeals to a theorem of M. Hall [4]:

THEOREM 1.1. For a given n-tuple of elements $b_{1}, b_{2}, \ldots, b_{n}$ not necessarily distinct, in an abelian group $G$ of order $n$, a permutation

$$
\left(\begin{array}{llll}
a_{1} & a_{2} & \cdots & a_{n} \\
c_{1} & c_{2} & \cdots & c_{n}
\end{array}\right)
$$

of the elements of $G$ exists such that

$$
c_{i}-a_{i}=b_{i}, \quad 1 \leqslant i \leqslant n \text { : f and only if } \sum_{i=1}^{n} b_{i}=0 .
$$

Chang's proof is to show that, in all but a few exceptional cases, the elements of $V$ associated with an $n \times n$ partial latin square with prescribed diagonal can be assigned the elements of the additive abelian group $Z_{n}$ so that the sum of the elements on the main diagonal is 0 . Then, by Hall's theorem, this array is embeddable in the operation table for addition for $Z_{n}$ and this, of course, is a latin square. It is the purpose of this paper to show that when $n$ is a prime one can determine exactly the number of distinct ways in which this assignment can be made.

To be more precise, suppose that the $n \times n$ partial latin square $L$ with prescribed diagonal has $k$ distinct symbols $y_{1}, y_{2}, \ldots, y_{k}$ occurring on the main diagonal. Suppose $y_{i}$ occurs precisely $m_{i}$ times, $1 \leqslant i \leqslant k$. Clearly, $\sum m_{i}=n$. Theorem 1.1 guarantees that $L$ can be completed to a latin square if there exists a mapping $f: V \rightarrow Z_{n}$ such that

$$
\sum_{i=1}^{k} m_{i} f\left(y_{i}\right)=0
$$

We will assume that $m_{1} \leqslant m_{2} \leqslant \cdots \leqslant m_{k}$. The problem now is to show that there exist $k$ distinct elements $x_{1}, x_{2}, \ldots, x_{k}$ in $Z_{n}$ such that

$$
\sum_{i=1}^{k} m_{i} x_{i}=0 \quad(\bmod n)
$$

except in the case $k=2$ where such a solution is clearly impossible. In the next section, we enumerate the number of such solutions when $n$ is a prime number.

\section{Number of solutions}

Consider the equation

$$
\sum_{i=1}^{k} m_{i} x_{i}=0
$$


over $Z_{n}$ where $\sum_{i=1}^{k} m_{i}=0$ but $\sum_{i=1}^{l} m_{j_{i}} \neq 0$ for any $l, 1 \leqslant l \leqslant k-1$ and any $\left\{j_{1}, j_{2}, \ldots, j_{l}\right\} \subset\{1,2, \ldots, k\}$. A solution by distinct elements of $(1)$ is a solution $\left(y_{1}, y_{2}, \ldots, y_{k}\right)$ of $(1)$ in which $y_{i} \neq y_{j}$ for all $i, j, 1 \leqslant i \leqslant j \leqslant k$. Let $S(\bar{m})$ be the number of solutions by distinct elements of (1). We now state and prove the main theorem. The proof requires a basic familiarity with elementary graph theory. The reader is referred to Bondy and Murty [1] for definitions.

THEOREM 2.1. For $n=p$, a prime number

$$
S(\bar{m})=(k-1) !\left\{\left(\begin{array}{l}
p-1 \\
k-1
\end{array}\right)+(-1)^{k-1}(p-1)\right\} .
$$

Proof. We say that a solution $\left(x_{1}, \ldots, x_{k}\right)$ to $m_{1} x_{1}+\cdots+m_{k} x_{k}=0$ has property $P_{i j}$, for $1 \leqslant i \leqslant j \leqslant k$, if $x_{i}=x_{j}$. Then $S(\bar{m})$ is the number of solutions which have none of these $\left(\begin{array}{l}k \\ 2\end{array}\right)$ properties. Thus, by inclusion-exclusion, the required number is

$$
S(\bar{m})=\sum_{l=0}^{\left(\frac{k}{2}\right)}(-1)^{l} \sum_{\substack{\alpha \subseteq \theta_{k} \\|\alpha|=l}} N(\alpha)
$$

where $\theta_{k}=\{(i, j): 1 \leqslant i \leqslant j \leqslant k\}$, and, if $\alpha=\left\{\left(i_{1}, j_{1}\right), \ldots,\left(i_{l}, j_{l}\right)\right\}, N(\alpha)$ is the number of solutions in which $x_{i_{1}}=x_{j_{1}}, \ldots, x_{i_{i}}=x_{j_{i}}$.

We determine $N(\alpha)$ by considering the graph $G(\alpha)$, on labelled vertices $\{1,2, \ldots, k\}$ and edge set $\alpha$. If $G(\alpha)$ has $c$ connected components, then $N(\alpha)=$ $p^{c-1}$ if $c>1$, and $N(\alpha)=p$ if $c=1$. This is seen most easily by considering two examples.

If $k=6$ and $\alpha=\{(1,3),(2,3),(4,5)\}$, then $G(\alpha)$ has 3 connected components, on vertex sets $\{1,2,3\},\{4,5\}$ and $\{6\}$. Moreover, $N(\alpha)$ is the number of solutions in which $x_{1}=x_{2}=x_{3}=x_{123}$ and $x_{4}=x_{5}=x_{45}$. Thus $N(\alpha)$ is the number of solutions in $G F(p)$ to

$$
\left(m_{1}+m_{2}+m_{3}\right) x_{123}+\left(m_{4}+m_{5}\right) x_{45}+m_{6} x_{6}=0 .
$$

But none of $m_{1}+m_{2}+m_{3}, m_{4}+m_{5}$, and $m_{6}$ are equal to 0 , so we let $x_{123}$ and $x_{45}$ take on any value in $G F(p)$ and can then determine $x_{6}$ uniquely, since $m_{6}^{-1}$ exists. Thus, $N(\alpha)=p^{2}$ in this case.

If $k=4$ and $\alpha=\{(1,2),(1,4),(2,4),(3,4)\}$, then $G(\alpha)$ is a connected graph, and thus has a single connected component. In this case $N(\alpha)$ is the number of solutions in which $x_{1}=x_{2}=x_{3}=x_{4}=x_{1234}$, and is thus the number of solutions in $G F(p)$ to

$$
\left(m_{1}+m_{2}+m_{3}+m_{4}\right) x_{1234}=0 .
$$


But $m_{1}+m_{2}+m_{3}+m_{4}=0$, and so $x_{1234}$ can be any element of $G F(p)$. Accordingly, in this case, $N(\alpha)=p$.

Thus

$$
S(\bar{m})=\sum_{l=0}^{\left(\frac{k}{2}\right)}(-1)^{l} \sum_{c=1}^{k} p^{c-1+\delta_{c, l}} g_{k}(c, l),
$$

where $g_{k}(c, l)$ is the number of simple graphs on $k$ labelled vertices, with $c$ components and $l$ edges. The generating function for these numbers is well-known (see, for example, [3]), and is given by

$$
\sum_{k \geqslant 0} \sum_{l=0}^{\left(\frac{k}{2}\right)} \sum_{c=1}^{k} g_{k}(c, l) z^{c} y^{\prime} \frac{x^{k}}{k !}=\left\{\sum_{i \geqslant 0} \frac{x^{i}}{i !}(1+y)^{\left(i_{2}\right)}\right\}^{z} .
$$

Moreover

$$
\sum_{k \geqslant 0} \sum_{l=0}^{\left(\begin{array}{c}
k \\
2
\end{array}\right)} g_{k}(1, l) y^{\prime} \frac{x^{k}}{k !}=\log \left\{\sum_{i \geqslant 0} \frac{x^{i}}{i !}(1+y)^{(i)}\right\} .
$$

Thus, using the notation $\left[x^{n}\right] f(x)$ to denote the coefficient of $x^{n}$ in the power series expansion of $f(x)$, we have

$$
\begin{aligned}
S(\bar{m}) & =p^{-1} \sum_{l=0}^{\left(\begin{array}{c}
k \\
2
\end{array}\right)} \sum_{c=1}^{k} g_{k}(c, l) p^{c}(-1)^{l}+(p-1) \sum_{l=0}^{\left(\begin{array}{c}
k \\
2
\end{array}\right)} g_{k}(1, l)(-1)^{l} \\
& =\left[\frac{x^{k}}{k !}\right]\left\{p^{-1}\left\{\sum_{i \geqslant 0} \frac{x^{i}}{i !}(1-1)^{\left(\frac{1}{2}\right)}\right\}^{p}+(p-1) \log \left\{\sum_{i \geqslant 0} \frac{x^{i}}{i !}(1-1)^{\left(\frac{1}{2}\right)}\right\}\right\} \\
& =\left[\frac{x^{k}}{k !}\right]\left\{p^{-1}(1+x)^{p}+(p-1) \log (1+x)\right\} \\
& =k !\left\{p^{-1}\left(\begin{array}{l}
p \\
k
\end{array}\right)-(p-1) \frac{(-1)^{k}}{k}\right\} \\
& =(k-1) !\left\{\left(\begin{array}{l}
p-1 \\
k-1
\end{array}\right)+(p-1)(-1)^{k}\right\} .
\end{aligned}
$$

\section{Conclusion}

To solve equation (1) of Section 2 over $Z_{n}$ when $n$ is composite appears to be much more difficult. As can be seen above, $S(\bar{m})$ is independent of the coefficients in (1) when the equation is over $Z_{p}$. For $n$ composite this is not the case. For example, the equations $x_{1}+2 x_{2}+3 x_{3}=0$ and $2 x_{1}+2 x_{2}+2 x_{3}=0$ over $Z_{6}$ have different numbers of solutions. 


\section{References}

[1] J. A. Bondy and U. S. R. Murty, Graph theory with applications (Amer. Elsevier, 1976).

[2] Gerald J. Chang, 'Complete diagonals of latin squares', Canad. Math. Bull. 22 (1979), 477-481.

[3] E. N. Gilbert, 'Enumeration of labelled graphs', Canad. J. Math. 8 (1956), 405-411.

[4] M. Hall, Jr., 'A combinatorial problem on abelian groups', Proc. Amer. Math. Soc. 3 (1952), 584-587.

University of Waterloo

Waterloo, Ontario

Canada 